\title{
The Role of Varnish Fluoride in Preventing Tooth Decay in Young Children
}

\author{
Mohammad Karimi DMD, BS* \\ Department of Pediatric, Sepideh Dental Clinic, Iran
}

Received: 制: October 26, 2018; Published: 眥: November 02, 2018

*Corresponding author: Mohammad Karimi DMD, BS, Department of Pediatric, Sepideh Dental Clinic, Iran

\section{Introduction}

Dental caries is one of the most common diseases that occur among the segments of society with any race, gender, age and social status. Fewer people can be found in the community without decayed or restored teeth. For many years, dental science has shown the effect of fluoride as a preventative agent of dental caries. Perhaps the greatest contribution to improving the dental health of children around the world is the discovery and use of fluoride as a deterrent to decay. Some decayed permanent teeth can be caused by initial injuries, just when the enamel has not yet been completely rigid. Since oral dentistry is difficult and challenging for minors, using Varnish fluoride can be a more effortlessly and effective solution. Varnish Fluoride is the newest therapeutic treatment for the prevention of tooth decay in different ages. This high flavored gel with a hearty aroma greatly increases the strength of the tooth and strengthens its enamel.

\section{Fluoride Action Mechanism}

Fluoride is a mineral that is found in nature and in many foods such as tea, water, seafood, vegetable types, and so on. This material accumulates in the structure of some tissues of the body, such as bones and teeth. The meaning of the varnish is lacquer, and it refers to the use of this material on the surface of the tooth that resembles the nail polish. It is among all forms of fluoride with the highest concentration of fluoride. At the same time, it's very safe and easy to use. Varnish Fluoride is a thick liquid that is rubbed as a protective coating on the teeth surfaces. Fluoride blocks the activity of acid-producing bacteria and protects teeth against sugar, bacteria, plaque, and acid and prevents tooth decay. Teeth that are in the early stages of decay are usually well treated with Varnish Fluoride. Fluoride also acts on oral microorganisms and reduces acid production by these microorganisms. On the other hand, fluoride enters the enamel of newly developed teeth and increases the mineralization (the maturity of the enamel) and reduces the vulnerability to decay. Fluoride is not only effective in preventing a new lesion in the teeth, but it is important to stop or at least to slow down the progression of primary caries lesions on the tooth surface.

\section{Complications of Fluoride Loss}
a) Having gum disease
b) Tooth Decay
c) Tooth loss
d) Cancer of the mouth and throat
e) Feeling pain in the face and mouth
f) Oral ulcers and infections
g) Other disorders and diseases that adversely affect chewing, eating, talking, smiling, mental and social health.

\section{Advantages}

Varnishes fluoride can be considered an amazing development in dentistry. Here are some of the benefits of using varnish fluoride:

a) Have a pleasant taste and a different essence.

b) Despite the flowing saliva in the mouth, it rapidly dries out.

c) For people who do respond quickly when something goes in their mouths or feel nausea, do not cause any problems. Usually, varnish fluoride does not require a dental tray.

d) Reduce the risk of sugar bacteria.

e) Fast and easy to do.

f) Does not require much dental equipment.

g) Due to their fast bonding properties, they stick to the teeth and have a high durability and stability. 
h) Poisoning caused by fluoride and its compounds, even if swallowed, does not threaten the patient.

i) Fluoride concentration in varnish is more than gel and foam.

j) Because a very little fluoride is used in this treatment, it might result in swallowing a small amount of it which causes no hazard for the patient.

k) Decrease in teeth decay, comfortable and acceptable use, safe, good and suitable method for mentally and physically disabled children is the advantages of using varnish fluoride for this group.

\section{Side Effects of Varnish Fluoride in Adults}

Many experts believe that if fluoride treatment is performed properly, no complications would endanger the patient, but if it is not done correctly, the side effects are usually very small and minor. The color of the teeth is slightly altered due to the adhesion and color of the varnish and tends to be yellowish. However, this mode of color change is entirely temporary. The varnish is removed from the tooth surface over time by brushing the teeth, and the resulting yellow color will disappear.

\section{The Other Side Effects of Fluoride Compounds Include}

a. Formation of white patches in the tooth that only the dentist will notice.

b. Vomiting, nausea and abdominal pain.

c. Allergic pimples.

d. Mouth ulcers.

\section{Who Does Need the Varnish Fluoride Treatment?}

This type of treatment is not only applied to teeth in children but also adults can benefit from varnish fluoride. The best applicants for fluoride therapy are:

a. People who live in the areas where the fluoride in drinking water is lower than the standard level

b. Children who have a high risk of dental caries

c. Children who use the bottle of milk

d. People with a family history of dental caries

e. Mentally and physically retarded people

f. People undergoing radiotherapy in the neck region

g. Children who under the orthodontic treatment

h. People who have healthy teeth but need to have preventive dentistry

i. Very young children or non-cooperative children

j. Children under 3 years of age who have no sources for local fluoride

k. People who have teeth that are sensitive. l. People who because of their gum and root toothache, are more likely to develop caries.

m. People who are indifferent to brushing and flossing

n. Those that their mouths are dry.

o. Those that drink a lot of acidic drinks.

p. People who attempted to fill teeth several times.

q. Those that the growth of their teeth is associated with a problem.

\section{Varnish Fluoride Intake}

Varnish fluoride treatment is done by the dentist at the office. It should be noted that the fluoride concentration used in this treatment is very high and it cannot be given without a prescription. The dentist places this viscous material directly on the patient's teeth and it attaches completely to the teeth. Varnish fluoride will be cleaned from the teeth after several hours. Although only 5\% of the fluoride concentration is used in this type of treatment, fluoride is present in other compounds, such as drinking water containing fluoride, fluoride gel, mouthwash and toothpaste containing concentrated fluoride. Varnishes fluoride compounds usually contain a solvent, resin, sodium fluoride and essences, and will rapidly dry.

\section{Important Considerations Before Using Varnish Fluoride}

When deciding on fluoride therapy, the considerations of these items in this section will be necessary.

\section{a. Crown of Teeth}

The crown of the perforated and decayed teeth, damaged teeth, teeth wear and cervical caries cause teeth sensitivities and damages. In this case, damaged areas of the tooth are more resistant to fluoride treatment.

\section{b. Age}

When patients get older, they are more likely to develop gum disease. When they are 50 years old, the roots of their teeth are more likely to develop carious lesions. Therefore, the treatment of Varnish Fluoride as an obstacle to the root caries, or at least minimizes the rate of root destruction. The treatment is effective and the prognosis is good.

\section{c. Restoration of Dental Caries}

The process of dental caries is very slow. Make sure the teeth are restored properly. If tooth decay is not deep and so-called is in the "early stages", it can be treated, or minimize caries with fluoride varnish.

\section{Treatment}

Topical treatment of fluoride therapy should be part of every precautionary care program, including the teaching of brushing techniques, regular dental examinations, and dietary control. Before the treatment, the dentist may advise eating common foods 
and drinks, but at least 2 days after treatment, the patient should avoid taking fluoride supplements. The concentration of fluoride available for treatment is far higher than the concentration of fluoride in the toothpaste and oral mouthwashes that we use every day. Varnish Fluoride is done with the help of a small brush or cotton swabs and the procedure does not last for more than a few minutes. The varnish is applied to the teeth surfaces for a few minutes and in some rare cases for several hours, then fluoride should be spreading to areas that need more substance. If the patient has dental sensitivity repeatedly, the procedure may be repeated every 6 months, as recommended by the dentist.

This is done in dental offices with different types of fluoride that can be used as gel, foam or materials called "varnish". The dentist will place the material on the teeth surfaces. Usually, the gels and the foams are placed inside the mouth with special trays and must remain for 4 minutes, but the varnishes are simply applied to the teeth with tools such as a brush or a cotton swab. Doing this does not require any kind of dental anesthesia and is not painful for the children. So, the kid would not have any stress or anxiety during the dental procedure. Most fluorides with different flavors that are not annoying to the kids are made. Choosing the type of fluoride therapy is a dentist's discretion. Usually, the dentist determines the fluoride therapy interval according to the condition of the teeth. The intervals range for most children is from 4 to 6 months.

\section{Post Treatment care}

a. According to the dentist's recommendation, at least 30 minutes after treatment, refrain from eating and drinking so that fluoride is absorbed into the teeth and can treat the decayed areas.

b. The treatment of varnish fluoride depends on the condition of the patient's oral health, and the dentist may need to re-treat the patient every 3 months, every 6 months or even once a year.

c. After application of Varnish Fluoride for children, in addition to not let them to brush their teeth until one day, they should avoid eating for 2 to 3 hours, and especially using solid foods until the end of the day.

d. It is also reminded that the teeth of children may change to yellowish and opaque which is temporary and will return to the color that was on the first day. A minor change in the color of the teeth after the treatment is completely normal and temporary, so parents should not be worried about this.

\section{Difference Between Topical and Systemic Fluoride Absorption}

If fluoride is ingested orally from various sources, it flows through the intestinal tract and then accumulated in the bones and teeth, or it is excreted through the kidneys. This process is called "systemic fluoride absorption". In the use of fluoride where only are applied on the teeth, the mechanism of action is different. In this case, fluoride prevents the loss of minerals on the surface of the teeth, and if the onset of decay occurs in places of enamel, fluoride prevents decay by re-mineralizing them.

\section{A Few Tips on the Importance of Fluoride}

a. It is imperative for everyone to use fluoride-containing toothpaste at home. Since the risk of caries in the adult teeth is far more than that of children and adolescents, as a result, these people may require more fluoride treatments such as Varnish Fluoride.

b. The amount of fluoride in varnish fluoride is higher than fluoride in water, toothpaste, and mouthwashes. Most dentists recommend varnish fluoride to patients periodically.

c. Kinds of toothpaste contain fluoride, but this fluoride alone is not enough to prevent caries from decayed teeth, and it is better for the dentist to perform fluoride therapy according to the child's condition.

d. Local application of fluoride in dental offices is recommended for all children and adolescents. Even if a child does not have tooth decay, topical fluoride is recommended as a means to increase the amount of fluoride in newly developed enamel and increase the resistance of these teeth to decay.

e. Fluoride therapy is also effective for adults. Because varnish fluoride is not only effective in preventing a new lesion but also it is important to stop or at least to slow down the progression of primary carious lesions on the tooth surfaces.

f. Fluoride-containing mouthwashes are recommended only for children over 6 years of age. Most of these mouthwashes contain sodium fluoride. Proper parent monitoring is effective in preventing tooth decay when using the mouthwash by the child. It is also important to train the child to spit mouthwash out of the mouth.

g. In fluoride therapy, a dental clinic uses fluoride to a certain extent and in accordance with certain principles, so a minimum of fluoride is swallowed and there is no possibility of poisoning. Fluoride intoxication in systemic uses is caused by very high levels of ingestion, which is very rare.

\section{Fluoride Therapy Implementation Plan in Iran}

Fluoride therapy is one of the most suitable methods for preventing and reducing decay in teeth in children. In recent years, the Ministry of Health has implemented the plan for children's fluoride therapy in primary schools in the country. The problems of dental caries in adults are gradually formed from the time of childhood, but according to the head of the oral health department at the Ministry of Health, the rate of referral to the dentist in childhood is very low. He also mentioned that with the implementation of this national and targeted program, a reduction of dental caries should be seen in elementary school students, and referral to oral hygiene centers for routine dental examinations will increase. ; Of course, reaching it is very difficult to achieve. Another of these goals is the stress reduction in children. Most children are afraid of visiting a 
dental office, and sometimes they have bitter memories of dental experiences, therefore, the painlessness of doing Varnish fluoride can reduce the fear of children and give them happy memories of dentistry.

\section{What is Dental Fluorosis?}

If at the time of formation of the teeth, the person is in an environment where the drinking water has fluoride content higher than the limit, the tooth receives high fluoride through the bloodstream and this amount changes the composition of the enamel crystals, therefore, the tooth would be shaped like gypsum or brown spots. This condition is commonly seen in all teeth that are exposed to high fluoride in drinking water. So, there may be a number of teeth or all the teeth of this person have fluorosis. We should assure parents that fluorosis will not occur with topical fluoride therapy in a dental office.

\section{Last word}

The results of the study indicate that the use of Varnish fluoride reduces the incidence of early caries symptoms and prevents its progress. However, the side effects of this study, such as the role of fluoride varnish in abnormalities such as a toothache and dental abscess shows no definitive announcement has been made so far.

\section{References}

1. Hunter JW, Chan JT, Featherstone DB (2006) Professionally applied topical fluoride: Evidence-based clinical recommendations. J Am Dent Assoc 137(8): 1151-1159.

2. Weintraub JA, Ramos Gomez F, Jue B (2006) Fluoride varnish efficacy in preventing early childhood caries. J Dent Res: 85(2): 172-176.

3. (2006) Carranza's Clinical Periodontology ( $\left.10^{\text {th }} \mathrm{edn}\right)$.

ISSN: 2574-1241

DOI: $10.26717 / B J S T R .2018 .10 .001996$

Mohammad Karimi. Biomed J Sci \& Tech Res

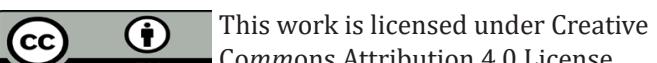

Submission Link: https://biomedres.us/submit-manuscript.php
4. (2008) Hand book of Pediatric Dentistry ( $3^{\text {rd }}$ edn). In: Angus C Cameron, Richard pwidmer (Eds.) Mosby Elesvier.

5. (2006) Promoting Children's Oral Health - Theory and Practice. Aubrey Sheiham, Marcelo Bonecker (Eds.).

6. (2009) Primary Preventive Dentistry. Norman O Harris, Franklin Garcia Godoy, Christine Nielsen.

7. Blanaid Daly, Richard G Watt, Paul Bachelor, Elizabeth T Treasure (2007) Essential Dental Public Health.

8. Carole A palmer (2003) Diet and Nutrition in Oral Health.

9. (1998) Judi Ratliff Pavis, Cynthia A Stegeman, The Dental Hygienist's Guide to Nutritional Care.

10. Adair SM (2006) Evidence-based use of fluoride in contemporary pediatric dental practice. Pediatr Dent 28(2): 133-142.

11. (2012) American Academy of Pediatric Dentistry. Guideline on Fluoride Therapy. American Academy of Pediatric Dentistry Reference Manual. Clinical Guidelines. 34(6): 162-165.

12. Centers for Disease Control and Prevention (2001) Recommendations for using fluoride to prevent and control dental caries in the United States. MMWR 50(RR-14): 1-42.

13. Autio Gold J (2008) Recommendations for fluoride varnish use in caries management. Dent Today 27(1): 64-67.

14. Bawden JW (1998) Fluoride varnish: a useful new tool for public health dentistry. J Public Health Dent 58(4): 266-269.

15. Valeria CC Marinho, Lee Yee Chong, Helen V Worthington, Tanya Walsh (2016) Fluoride mouthrinses for preventing dental caries in children and adolescents. Cochrane Database of Systematic Reviews (3): CD002284.

16. Valeria CC Marinho, Helen V Worthington, Tanya Walsh, Lee Yee Chong (2015) Fluoride gels for preventing dental caries in children and adolescents. Cochrane Database of Systematic Reviews 15(6).

17. VCC Marinho (2008) Evidence-based Effectiveness of Topical Fluorides. Advances in Dental Research 20(1): 3-7.

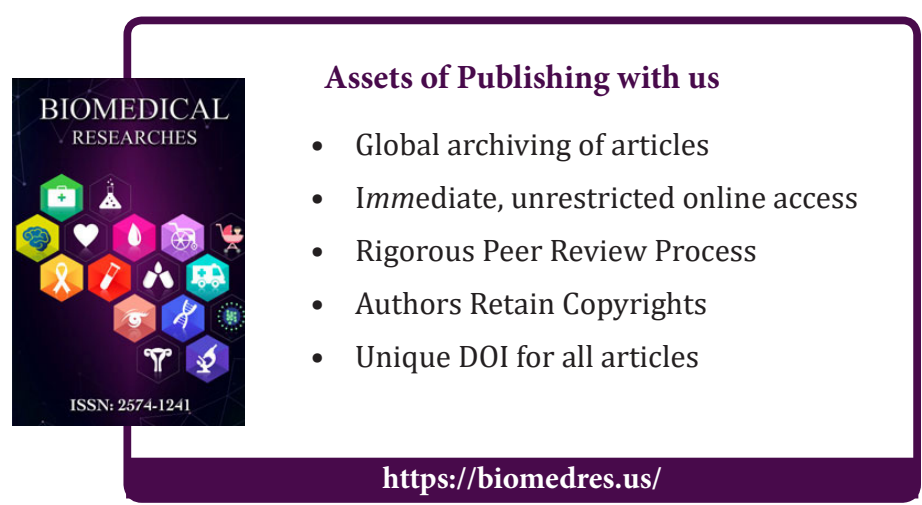

